\title{
DrugMetZ DB: an anthology of human drug metabolizing Chytochrome P450 enzymes
}

\author{
Tresa Remya Thomas Antony and Shanthi Nagarajan* \\ Bioinformatics Centre, Pondicherry University, Pondicherry - 605 014; India; \\ Shanthi Nagarajan * - Email: shanthi_bi@linuxmail.org; Phone: +91 413 2655212; Fax: +91 413 2655211; \\ * Corresponding author; Both the authors contributed equally
}

received August 31, 2006; revised November 03, 2006; accepted November 09, 2006; published online November 14, 2006

\begin{abstract}
:
Understandings the basics of Cytochrome P450 (P450 or CYP) will help to discern drug metabolism. CYP, a super-family of heme-thiolate proteins, are found in almost all living organisms and is involved in the biotransformation of a diverse range of xenobiotics, therapeutic drugs and toxins. Here, we describe DrugMetZ DB, a database for CYP metabolizing drugs. The DB is implemented in MySQL, PHP and HTML.
\end{abstract}

Keywords: Cytochrome P450; drug metabolizing enzymes; bioinformatics

Availability: www.bicpu.edu.in/DrugMetZDB/

\section{Background:}

Cytochrome P450 (P450 or CYP), a superfamily of hemethiolate proteins, are found in almost all living organisms and are involved in the biotransformation of a diverse range of xenobiotics, including therapeutic drugs and countless toxins, and physiologically important hormones such as steroids, arachidonic acid, bile acids, and retinoic acid. [9] For instance cytochrome P450 CYP2C9 subtype is responsible for the metabolism of S-warfarin. [3] The basic purpose of drug metabolism in the body is to make drugs more water soluble and thus more readily excreted in the urine or bile. One common way of metabolizing drugs involves the alteration of functional groups on the parent molecule (e.g., oxidation) via the cytochrome P450 enzymes. [8]

Pharmacokinetics and drug metabolism have been shown to be of greater importance during drug development today. It is evident that drugs that are too rapidly metabolized by drug-metabolizing enzymes are mainly localized in the liver. [5] An evaluation of the mechanism for the metabolic clearance of 315 different drugs revealed that $56 \%$ of them were primarily cleared through the action of the cytochrome P450 enzymes, with CYP3A4 being by far the most important (50\%) followed by CYP2D6 (20\%), CYP2C9/19 (15\%), and the remaining metabolism carried out by CYP2E1, CYP2A6, CYP1A2, and unidentified P450s. [1] The CYP mixed function monooxygenases are located on the smooth endoplasmic reticulum of cells throughout the body especially in the liver. [2] A major obstacle for the drug industry today is, however, the extensive interindividual variation in human drug metabolism. [4] The main causes for the variation observed in drug metabolism are the genetic differences. [5] These

ISSN 0973-2063

Bioinformation 1(7): 248-250 (2006) differences are called genetic polymorphism's and are linked to inherited autosomal recessive traits. [6] There are different isozymes of CYP, arised due to genetic variations which governs the metabolism of different categories of drugs. Hence, a database on drug and corresponding CYP isozymes is described.

\section{Methodology:}

Data collection and storage

Database consists of data for different category of CYP isozymes and metabolizing drugs. CYP related data such as metabolizing drug, tissue specificity, cellular location and gene ID were collected from published literatures [reference available at every entry]. In addition, CYP protein sequences from Swissprot [10] were also collected. The IUPAC name, chemical formula, smile string, drug description, etc. were collected from DrugBank [7] for CYP associated drugs.

MyISAM type of engine for MySQL database system is used to store data. This particular engine manages non transactional tables and allows high speed storage and fast retrieval of its data through a non-clustered index and data organization. At present DrugMetZ DB covers 131 drugs and 12 subtypes of CYP isozymes.

\section{Database design and interface}

Tables were created with different data types to hold dissimilar data. Each table was associated by means of primary key and foreign key to form a comprehensive data transaction. Validated data sets were stored under tables which are in relational database management system. PHP is used as an interface between HTML front end and 


\section{Bioinformation}

\section{www.bioinformation.net}

Web Database

MySQL back end to fetch data from database for user friendly display.

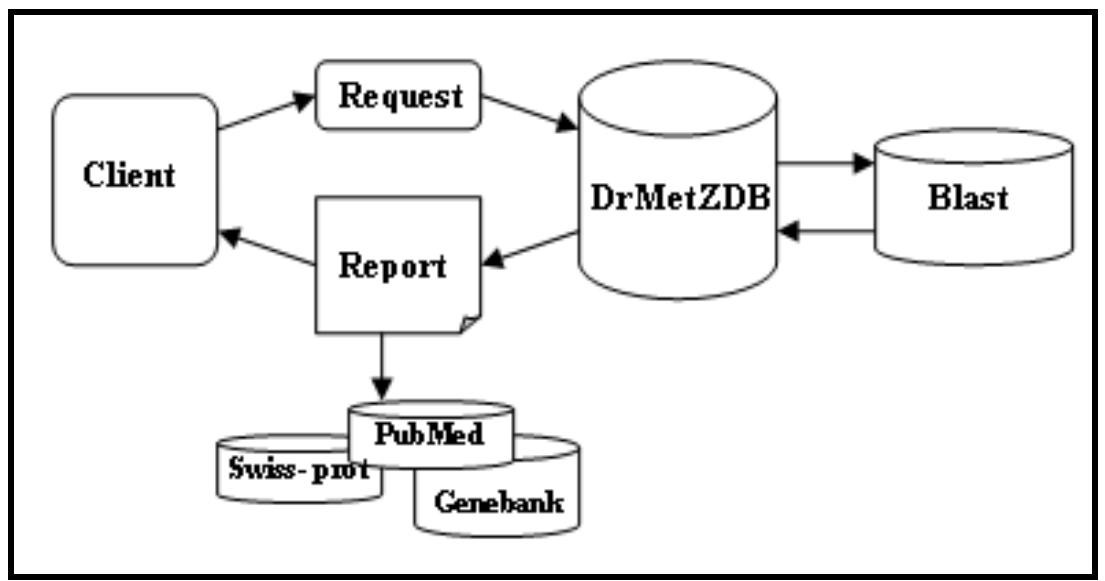

Figure 1: Over all process of database
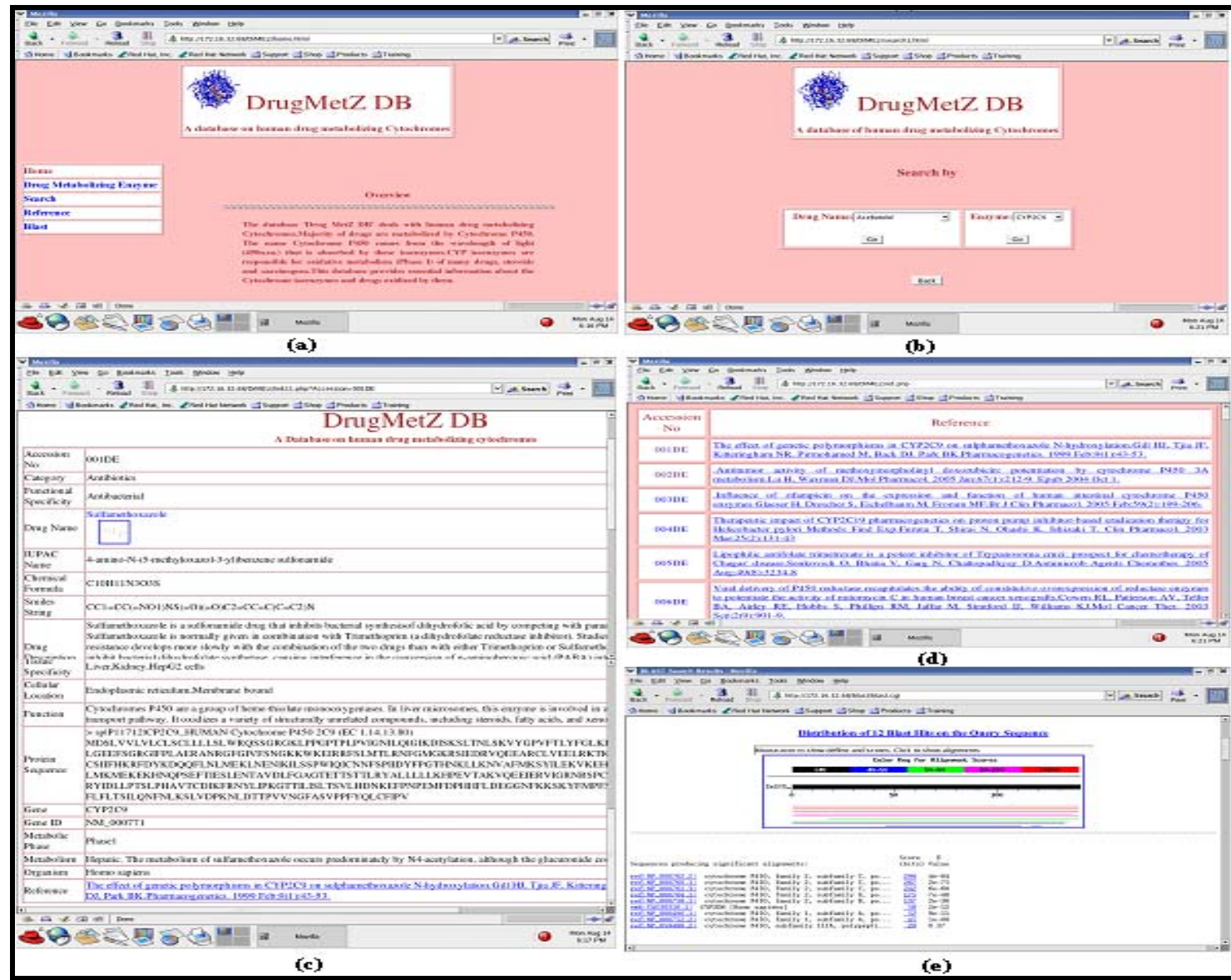

(c)

(e)

Figure 2: (a) Home page of DrMetZ DB (b) Shows search criteria (c) Report of Drug (d) Reference display with it's PubMed link (e) BLAST search result against Cytochrome P450 database

ISSN 0973-2063

Bioinformation 1(7): 248-250 (2006) 


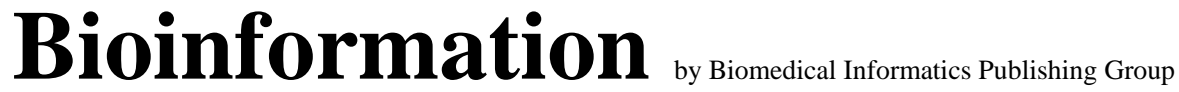

open access

\section{www.bioinformation.net}

Web Database

\begin{abstract}
Utility:
This database provides essential information about different categories of drugs and corresponding metabolizing. Cytochromal isozymes present in human body. It is a user friendly database, allows user to search for various drugs and enzymes. It displays relevant information about drugs, especially their chemical nature. In case of enzymes, it gives functional, genetic and protein level information. DrugMetZ DB database also gives link to major databases such as NCBI, Swissprot, RXLIST and related articles in pubmed. Blast search help clients to find sequence hits in Cytochrome P450 database. Key feature of this database provides specialized information on CYP, whereas databases like DrugBank [7], PharmGKB [12], ADME-AP [13], and KEGG [14] is generalized for drugs, enzymes, properties of drugs or relationship among drugs and diseases, respectively.
\end{abstract}

\section{Conclusion:}

Datasets held in this database collected from published literatures and other databases contains information for drugs, nucleotide and protein sequences.

\section{Future development:}

Our future update will include addition of inductors and inhibitors of CYP, linking with KEGG and to facilitate access to all human drug metabolizing CYP.

\section{Acknowledgement:}

The authors acknowledge Prof. P.P. Mathur, Coordinator, Bioinformatics Centre, Pondicherry University, India for constant encouragement and suggestion. Authors are also thankful to Department of Biotechnology, New Delhi, India for financial assistance.

\section{References:}

[01] R. J. Bertz \& G. R. Granneman, Clin Pharmacokinet., 32:210 (1997) [PMID: 9084960]

[02] M. L. Buck, Pediatric Pharmacotherap., 3 (1997)

[03] G. P. Aithal et al., Lancet, 27:353 (1999) [PMID: 10073515]

[04] J. Lazarou et al., JAMA, 279:1200 (1998) [PMID: 9555760]

[05] M. Ingelman-Sundberg, Drug Metab Dispos., 29:570 (2001) [PMID:11259354]

[06] H. E. Poulsen \& S. Loft, Arch Toxicol Suppl., 16:211 ( 1992) [PMID: 8192584]

[07] D. S. Wishart, et al., Nucleic Acids Res., 34:D668 (2006) [PMID : 16381955]

[08] M. J. Cupp \& T. S. Tracy, Am Fam Physician., 58:107 (1998) [PMID: 9447218]

[09] A. M. Yu, et al., Drug Metab Rev., 36:243 (2004) [PMID: 15237854]

[10] A. Bairoch, et al., Brief Bioinform., 5:39 (2004) [PMID: 15153305]

[11] S. F. Altschul, et al., Nucleic Acids Res., 25:3389 (1997) [PMID: 9254694]

[12] M. Hewett, et al., Nucleic Acids Res., 30:163 (2002) [PMID: 11752281]

[13] L. Z. Sun, et al., Bioinformatics, 18:1699 (2002) [PMID: 12490461]

[14] H. Ogata, et al., Nucleic Acids Res., 27:29 (1999) [PMID: 9847135]

Edited by P. Kangueane

Citation: Remya \& Nagarajan, Bioinformation 1(7): 248-250 (2006) License statement: This is an open-access article, which permits unrestricted use, distribution, and reproduction in any medium, for non-commercial purposes, provided the original author and source are credited. 